\title{
RESOURCE MANAGEMENT AND FAULT TOLERANCE IN SENSOR CLOUD
}

\author{
Prashant Sangulagi $^{1}$, Shivaleela C. Gudage ${ }^{2}$, A. V. Sutagundar ${ }^{3}$ \\ ${ }^{l}$ Department of Electronics and communication engineering, BKIT Bhalki India-585328 \\ ${ }^{2}$ Department of Electronics and communication engineering, BKIT Bhalki India-585328 \\ ${ }^{3}$ Department of Electronics and communication engineering, BEC Bagalkot India
}

\begin{abstract}
Nowadays all needs comfortable life like easy availability, safety, security, services can be fulfilled by cloud computing. For transferring the information into cloud accurately we used the sensor node. In the sensor-cloud there is a problem of managing of resources and detection of fault node. So that we propose a Resource management and fault tolerance in sensor-cloud. Here we do management of the resources without losing accuracy and fault nodes are detected and eliminated from the network without affecting the network. From this we increase network life time.
\end{abstract}

Keywords: cloud computing, sensor cloud, Resource Management, Fault tolerance, wireless sensor network, sensor node.

\section{INTRODUCTION}

\subsection{Wireless Sensor Networks}

Foremost wireless leaning is known as tone. wireless networks was formed in the 1980s. By 1999, it had started to connectionless information system. A connectionless sensor system can be specify as a arrangement of mechanism, indicated by holes, iwill intellect the atmosphere also converse the message collected by observed area throughout connectionless relations ${ }^{[1]}$. Statistics is promoted, probably abundant jumps, into drop will make employ of in the neighborhood in other words link by further nets, all the way from side to side a access. holes are stationary otherwise moving. They are conscious about position otherwise not. They can be identical or else different.

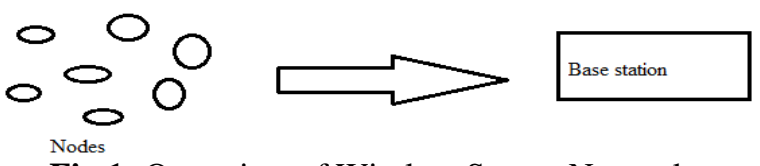

Fig 1: Over view of Wireless Sensor Network

\subsection{Cloud Computing}

Cloud computing is a mock-up, allow suitable, on require association right use mutual group of configurable compute message (such as complexs, attendants, collection, relevances and repairs) speedily gave and provided amid smallest organization attempt otherwise overhaul donor communication.

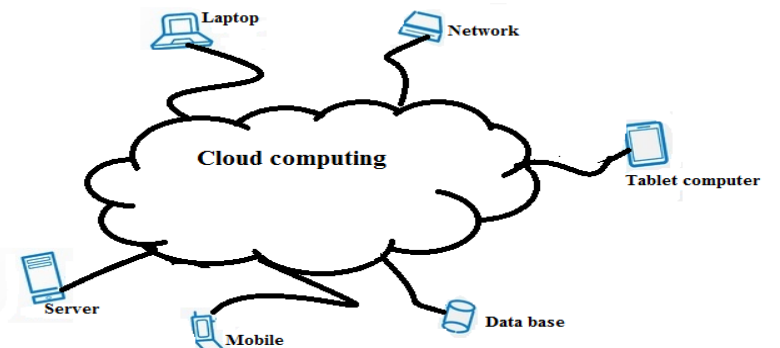

Fig 2: Cloud computing

\subsection{Sensor Cloud}

A single sensor data storage, visualization and remote administration stage, the dominant cloud computing technologies there build brilliant information scalability, fast visualization and programmable client study. initially is deliberate to bear long-standing connectionless antennas[2]. Sensor-cloud gathering and doling out of information from various sensor networks and acknowledge distribution of an huge scale data and cooperate with applications on the clouds [3]. The paper is organized as follows, section2 provide Literature survey. section 3 , provides sensor cloud communication. section 4 , provides resource administration in sensor cloud, section 5, provides fault tolerance in sensor cloud and section 6, proposed work and study about Resource management in sensor cloud environment, fault tolerance in sensor cloud environment and fault node recovery algorithm flow chart, Algorithm. section 7, discusses about graphs of performance parameter. Section 8 discusses the Conclusion.

\section{LITRATURE SURVEY}

Literature survey conceded out primarily for the investigation of the conditions of the modern assignment 
which support to observe the flaws in the existing organization. Confirmation on unanswered troubles cans effort. So, the next matter not only make clear the surroundings of the mission but also notice harms and deficiency that make them to put forward figuring out, and effort on this paper. A many of research has been ended on Resource management and fault tolerance in sensor cloud In the year 2015 chi yang[4], discovered Errors in huge amounts of message from sensor-power-plant group determined, a new approach will be bring forth, with cloud computing. to begin with it display error categories for bulky amounts of message following, the relationship between sensor networks, degree-gratis multifaceted arrangements are make known. For apiece kind of mistake and characteristic of degree-gratis arrangements beating out era capable stratagem in support of the discovery and place of flaw in great amounts of statistics on cloud. With the outcome of our customer cloud. Cloud-computing environment research has expressed.

a) Presented degree- gratis mistake observe concept crucial that may period decreases quick mistake noticed large statistics.

b) Submitted comparable choice error rate non-scale free error recognition advance proved.

Sudip Misra [5] set up a work provide a speculative mold of Virtualization for a sensor-cloud atmosphere. They exhibited a relative evaluation of the presentation among sensor cloud and WSN. Fallout provide that a cloud of feeler achieves recovered presentation measure up to a WSN in mainly events.. Here will be gostivid that the shift to the model of applications of technology based and lifetime to the sensor stand cloud beneficial in terms of presentation ease of make use, and gain. As cloud sensor inception arose, there is significant span for investigate.

Atif Alamri [6] Here, they studied the use of sensor-cloud structural design concerning to numerous applications. Sensor-Cloud design enables the sensor data is organize, collect, and routed, building it money-making, period offered and effortlessly easily reached. Previous were a good number WSN schemes, which integrated several manage/ordering compositions were connected to specific application of natural world zero or less interoperability, and non-expandable closed. We discussed the possibility designed for implementing technology to deal with added composite occurrence from the actual global through improvement capacity sensor service-cloud infrastructure.

Subasish Mohapatra, K. Smruti Rekha [7]' monitor the structure with cloud computing-monitor fitness and interchange of data among doctors, caretaker, to give canvassers by cloud to not expensive, first-class concern for operator get started sensors patients. Stretch in house atmosphere for the monitor and gathering of raw message. Upgrading in the prospective like the majority sensors and a number of further sensors can be deployed employ a microprocessor further powerfully and can boost cloud safety forever. You have to comprise other aspects such as organization consumer confirmation and approval structure

\section{SENSOR CLOUD}

Many organizations that are required to run businesses as well as job which only in cell phone. energetic and information culture surroundings education, distant check up, physical condition ,domicile securely machinery etc require unique communications that can supply nonstop data and secure mobile information, dependable plus adequate management system in a tight perimeter frame and users.

\subsection{What is a Sensor-Cloud?}

In as said with Intellisys cloud sensor can be defined as: "infrastructure grants report rampant really utilize the sensor interface among physical and cyber globe, and group of data-processing strength of character and the Internet as a means of contact ${ }^{[8]}$. Firstly considered to hold up continuing grow thing of wireless sensors. A cloud of sensor accumulates and processes message from numerous sensor networks agree to replace of data on a bulky level and the applications on the cloud for abusers to occupation jointly.

\subsection{Sensor-Cloud Structural Design}

Computing functioning employed to afford general network work which customer contemplate to be profit from the occupations and it is not regard with exhaustive mechanism services rendered. Involuntarily when the client needs proviso of service quickly (such as virtual sensor) generated by cloud computing services ${ }^{[9]}$

\subsubsection{Service Lifecycle Model of Sensor-Cloud}

System process can be splits into three phases. Before instantiating any preset part service, it is compulsory to set up the following:

(1) Preparation of information technology sources

(2) Preparation of corporal sensors.

(3) Preparation of repair model.

Customers can choose a mould suitable service and ask for the need work instances. These are utility instances involuntarily and at no cost to consumers that can then rapidly removed when it becomes hopeless. Single service guide you can make a numerous number of service instances. Normalized service make offered templates and service models can be joined when need by a variety of consumers. Ahead ${ }^{[10]}$, clock bringing together ${ }^{[11]}$, records processing [12], [13] energy management, OS [14], placements $^{[15]}$ concentrate little learn of physical sensors ${ }^{[16]}$. On the other hand, only a little studies are alert on physical sensor managing since these physical Sensors are directly line up with their particular application as well as for its precise customers directly involved but other users do not use these physical sensors as their parallel sensor services directly if essential.

Physical sensors are classified based on the sensor understandings as well as the real space from the occurrence. Investigate ${ }^{[17]}$ proposed a technique to set the sensor material errors of data assume the disparity among the rank space sensor message. 
on the other hand, the learn lead by the examiner objectives to evaluate the physical sensor faults, bond among implicit and corporeal sensors also implicit sensor and then present false outcome, pertinent corporeal sensor noticeable.

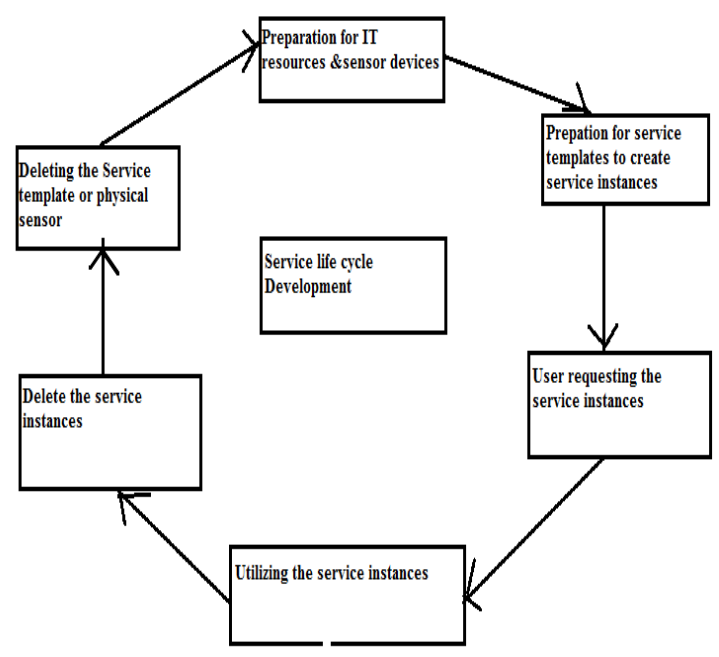

(a)

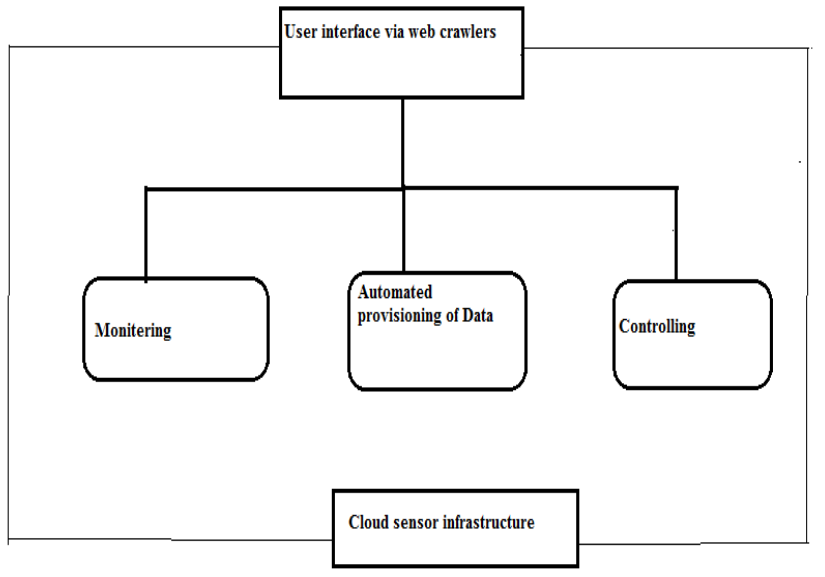

(b)

Fig 3(a \& b) : manifestation of Sensor-cloud time rotation expansion stage.

\section{RESOURCE MANAGEMENT IN SENSOR CLOUD}

In recent years, cloud computing ${ }^{[8]}$ come out as a dependable source, software and statistics on demand to give a novel IT pattern. Basically, sources supply cloud computing usable clients among implicit attendants.

Sensor negotiator cloud "virtualizes physical sensor node as the fail to pay" sensor operative ". Addicts can utilize and supervise sensor managers with standard occupations. Jointed sensor agents are given animatedly in reply to abuser demands.
The subsequent four characteristics of autonomic recognized the four basic areas of self-organization:

a) Arrange the Self goods: that agree to the scheme to acclimatize to changeable circumstances by mechanically altering the arrangement, such as addition or eliminate novel constituents or installation of software modifies without disruption of services by itself.

b) Self-heilende assets: which is to avoid and get better from fault by mechanically notice, identify, go arounding and make progressing from questions that can guide to service intermissions.

c) Optimization of Self nonstop itself, i.e., optimizes property, the scheme you can practically on subsisting processes and imprudent in answer to ecological circumstances get better.

d) Self-asset defense that notices, find, and protects adjacent to viruses and illegal access, rejection of service assaults. Self security and can also contain the capability to care for themselves from physical damage.

\subsection{Scheme Planning of Sensor Manager Cloud}

Organization construction enclosed "sensor operative cloud" in Figure 4. Below are explanations of the responsibilities of the main constituents.

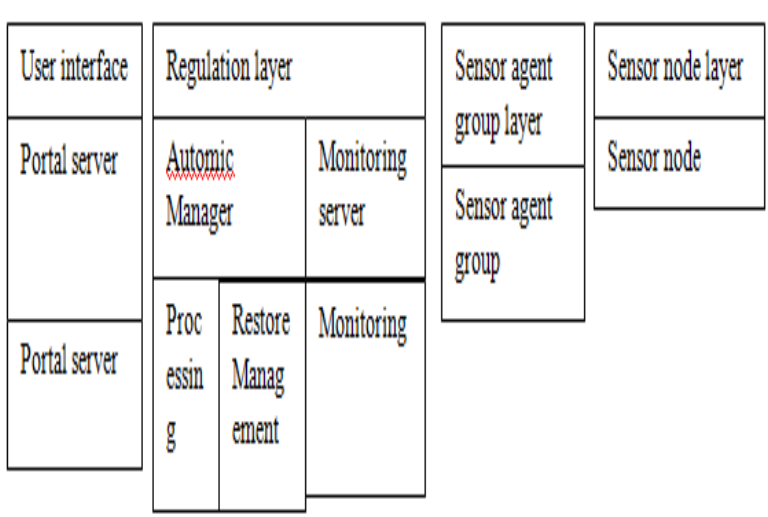

Fig 4: Scheme planning of Sensor mediator Cloud

\subsubsection{Porch Attendant}

Customer task-sensor node possessor or answerable for "sensor worker cloud" or customer, tells the functions obtainable to the consumer. For the buyer, the attendant demonstrates the access catalogs open, close, ask for to keep or obliterate the sensor managers working sets, supervising sensors, scrutinizing customers and make patterns for sensor manager assembles. For owners of a sensor node provides the set of choices to open, close portal, and record. Manager Superintendent offers the portal list of options to produce, modify and erase models for sensor mediator and sensor manager collections. And employ additional files illustrated that "cloud sensor machinist" to record or remove virtual attendants, handle customers and possessors detained the sensor, and ensure the position of your virtual servers. All files are obtainable to, customers the sensor node proprietors to be answerable for "sensor operative cloud." 


\subsubsection{Autonomic Director}

The independent supervisor convention sensor manager collections for access takes account of propagate locomotive.

\subsubsection{Sensor Manager Collection}

A sensor manager assembly is mechanically provided implicit attendant by director. All manager collection obsessed by buyer plus encloses single otherwise additional antenna managers. Customers can manage the sensor mediators.

\subsubsection{Supervising Attendant}

supervising attendant takes information concerning sensor managers through supervising mediators include implicit attendants. Accumulates, accepts information in folder.

\section{FAULT TOLERANCE IN SENSOR CLOUD}

Cloud contribution difficulty area sensor fault tolerance. Fault tolerance is a main apprehension to make sure the ease of use and dependability of very important repairs, as well as the implementation of the submission. To decrease the crash of fault scheme accomplishment and function, should failure prediction and error management and fault tolerance techniques used to forecast such non-performance and get appropriate act previous or behind a failure occurs. Fault node recovery (FNR) algorithm for growth the existence of a wireless sensor network (WSN). Sensor holes can guide plus use again extra direction-finding paths. So, the living of the WSN the algorithm not only enlarged but also decreases the price of interchanging the sensor node.

\subsection{Existance Work}

In 2004, Lee et al Pike. Four fundamental theoretical area allocated fault-tolerant schemes .conceptual note the fitness of person communications send and accepted. Theoretical mistake managing on the varieties of feasible faults as well as their belongings on the scheme. Mistake- hide from view conceptual "deal with neighboring report kinds to execute process on the facade mistakes. Lastly, the "theoretical" forms of details statistics and properties need for doing well communication mistakes.

In the year 2011, Arvind Kumar et. Al., discover dissimilar dependable mistake discovery and fault acceptance techniques. Their major focal point is on kinds of debt that utilizes fault discovery events and revival methods in the scheme this article includes, as these techniques be applied to perceive and to accept mistakes of dissimilar share out real-time schemes. The key aim of fault separation is connected to recognize the generate of the fault.

In 2013, Ravi et. al. And present inventive opinion, the scheme stage in the organization and administration of fault acceptance in the clouds. Comprehensive approach proposed sophisticated shadiness details mistake tolerance techniques for application developers and consumers during devoted service layer. Furthermore, it shows the scheme a) supplies a complete fault acceptance answer for consumer applications mixture choose fault acceptance machinery and (ii) make available the properties of an fault-acceptance answer using runtime supervising..

\section{PROPOSED WORK}

Our main objective of this Paper is

1) To detect and correct fault nodes at virtual network side and deletion as well as addition of node done at virtual network.

2) To monitor the changes in physical wireless sensor network through virtual wireless sensor network.

3) To preserve the scares resources and enhance the network life time.

4) To store information in priority wise at sensor cloud server.

5) To improve the overall efficiency of wireless sensor network

\subsection{Resource Management in Sensor Cloud Environment}

Resource management refers to the processes that are utilized to manage how capacities offered by cloud sources and give services to additional entity According to consumer obligations cloud supplier will give sources. If there are customers need the similar resource then managing sources, admin will provide resource both customers.

The below figure 6 shows the Public and private cloud. In public cloud the information can share anybody. In private cloud the information can be share by particular organization. On the both cloud we deployed a sensor node, the nodes are connected one another. From the sensor nodes the data will send to neighbor's node. From neighbor node data will sent to cluster Head. From that data will pass to Base station. From Base station data transmitted to Gateway. From the Gateway information sent to the sensor cloud. In the sensor cloud, the admin will manage the data. In the sensor, admin grouped. The grouping is based on priority. Higher priority, Normal priority, Lower Priority. In these grouped again sub grouped are Public cloud, Private cloud, Hybrid cloud. Here we were using Grid computing technique. As per user requirements Admin will send the data.

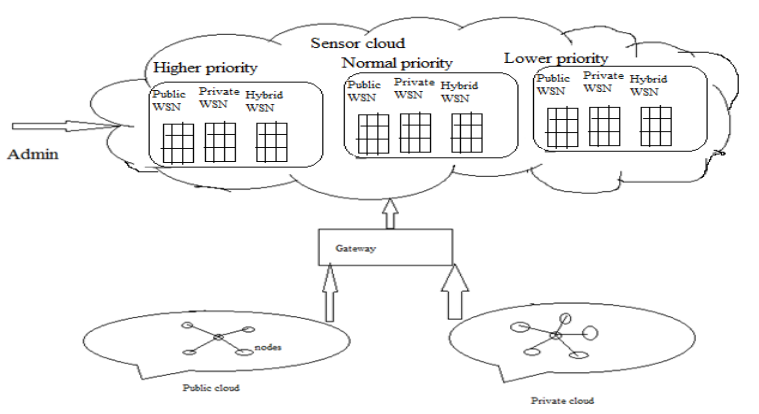

Fig 5: Resource management in sensor cloud 


\subsection{Fault Tolerance in Sensor Cloud Environment}

In sensor cloud the main difficulty area is fault acceptance. Fault acceptance is a main disquiet to assurance ease of use and dependability of vital jobs, relevance implementation.

The below figure 6 illustrates in the physical sensor network area we deployed sensor node, the nodes are connected one another. From the sensor nodes the data will send to neighbor node. From neighbor node data will pass to cluster Head. From cluster Head data send to Base station. From Base station data transmitted to Gateway. From the Gateway information sent to the sensor cloud. In the sensor cloud, the admin will manage the data. In the sensor cloud, admin will check that is it faulty node or not. If it is a faulty node then admin will remove that node form the sensor cloud. Admin will send message to physical sensor network that it is a faulty node. If it's not faulty, then admin will send the data to the required users.

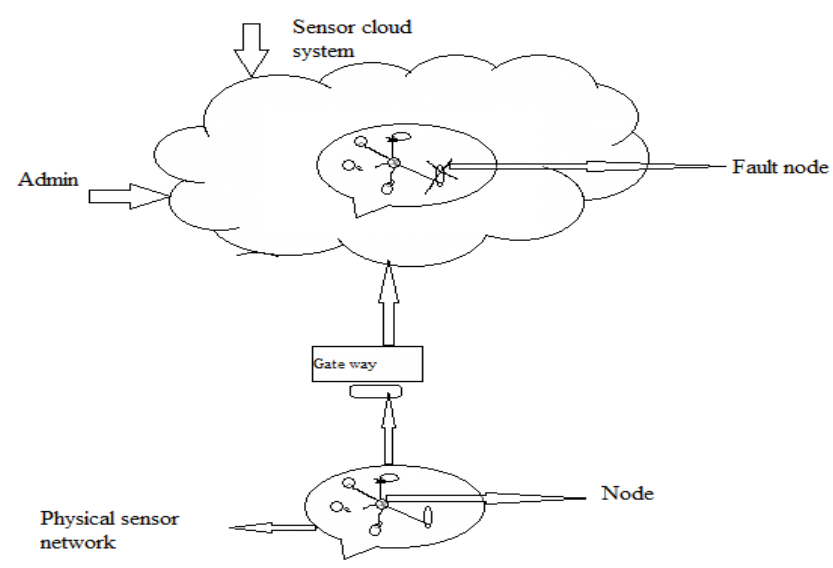

Fig 6: Fault tolerance in sensor cloud

\subsection{Fault Node Recovery Algorithm Flow Chart.}

A fault node recovery (FNR) algorithm for WSNs uses amount stretching algorithm mixed amid inherited algorithm. The FNR algorithm worth for every sensor hole with class allocation algorithm generates class worth, direction-finding table, near nodes, and consignment.

Figure 7 produces an mistake node retrieval algorithm flow chart Figure 5, FNR row value algorithm, the directionfinding table, the locate near holes, and all hole pack sensor price, with an algorithm make public.

There are 5 stages in the genetic algorithm: Initalization, Evaluation, choices, intersect, and alteration. as follows.

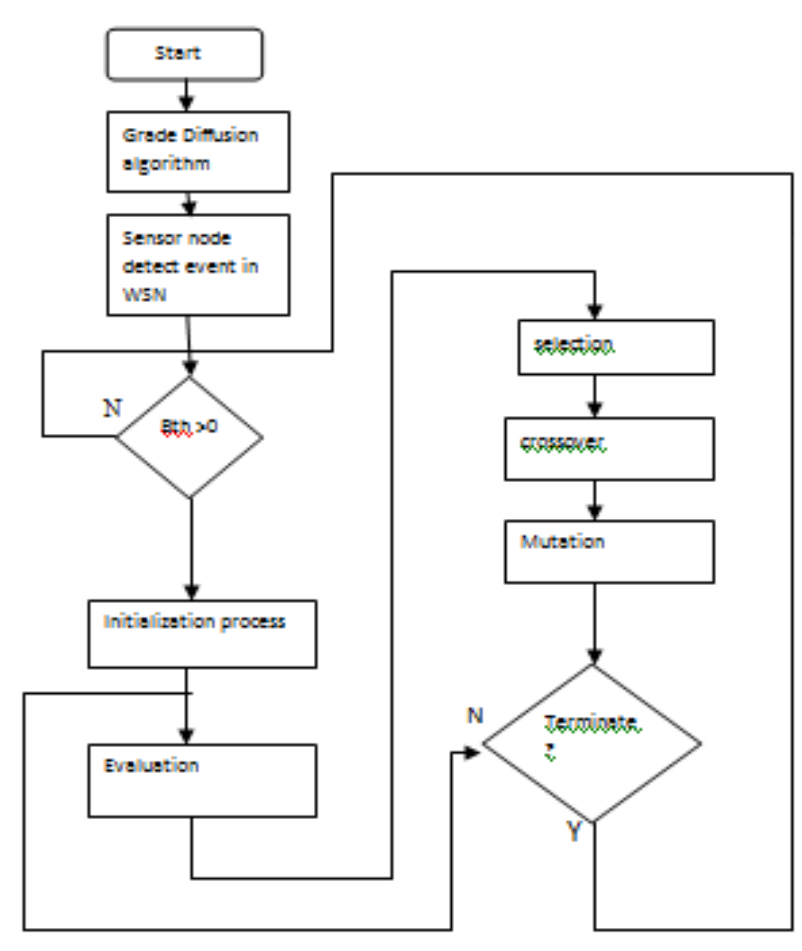

Fig 7: Fault node recovery algorithm flow chart.

\subsection{Algorithm}

\section{NOMENCLATURE:}

n: number of nodes

DD: Distributed diffusion algorithm

GD: Grade diffusion algorithm

FNR: Fault node recovery

- $\quad$ The network area is $200 * 200$.

- The number of nodes are ' $n$ '.

- The nodes are deployed randomly.

- $\quad$ Selecting some nodes as cluster head.

- Deploying Base station in network.

- If there is a fault node in the network found.

- For fault node recovery we use FIND algorithm.

- GD algorithm create direction-finding table, near node, payload rate for every sensor node.

- GD creates direction-finding for all sensor node and identifies.

- Sensor node send event data to the sink node according to GD algorithm.

- $\quad \mathrm{B}_{\text {th }}>0$, the algorithm invoke and replace nonfunctioning sensor Then algorithm will calculate the sensor node to exchange.

- Evolution is done where we find the fitness value.

- Selection eliminate the nodes with lowest fitness value.

- Keep the half of the nodes with better fitness value and put in the mating pool.

- The worst node is removed.

- In mutation step FNR algorithm will exchange the sensor nodes.

The terminate or stop the algorithm node by functional number 


\section{RESULTS}

\subsection{Initialization}

The below figure 8 illustrates, the network area having length $200 \mathrm{~m}$ and width is $200 \mathrm{~m}$. we deployed 50 sensor node randomly, here we initialization of sensor node done.

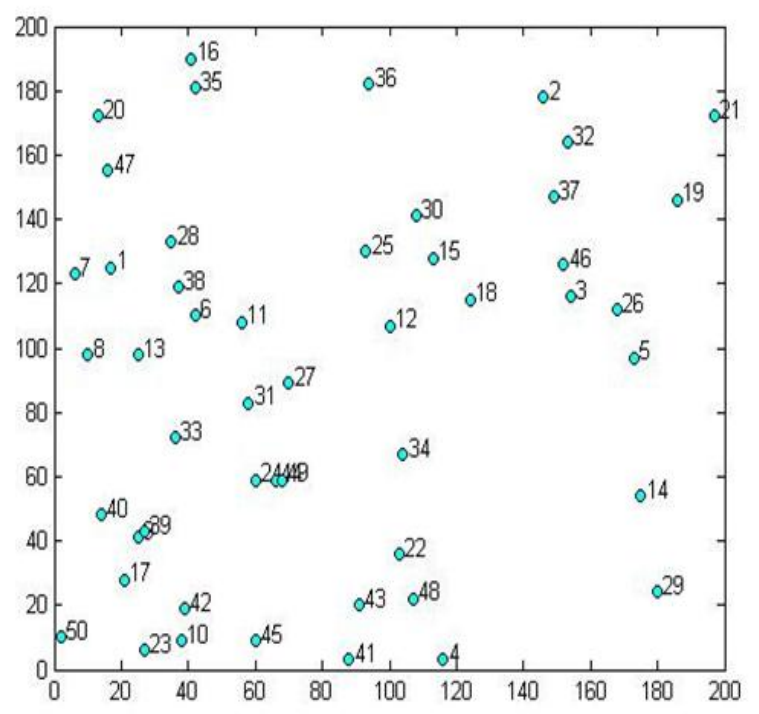

Fig 8: initialization of sensor nodes

\subsection{Selection}

In the figure 9 illustrates ,network area is about 200m length and $200 \mathrm{~m}$ width,50 sensor nodes are deployed .we grouped the sensor node called clusters, in this graph there are four clusters which are displayed with different color that is Blue, Yellow, Green, Red.

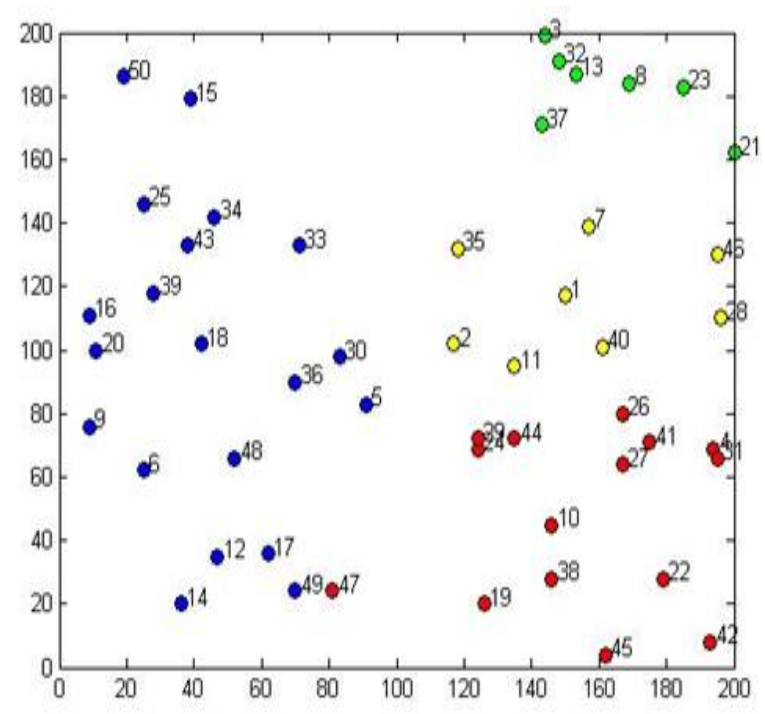

Fig 9: selection of clusters

\subsection{Deployment of Base Station and Clusters}

In figure 10 illustrates, network area is about $200 \mathrm{~m}$ length and $200 \mathrm{~m}$ width,50 sensor nodes are deployed. Here also nodes are grouped called cluster, here are four clusters. In each cluster one node considered as cluster head. One Base station is deployed. Sensor nodes will transmit information to cluster head, will transmit information to base station.

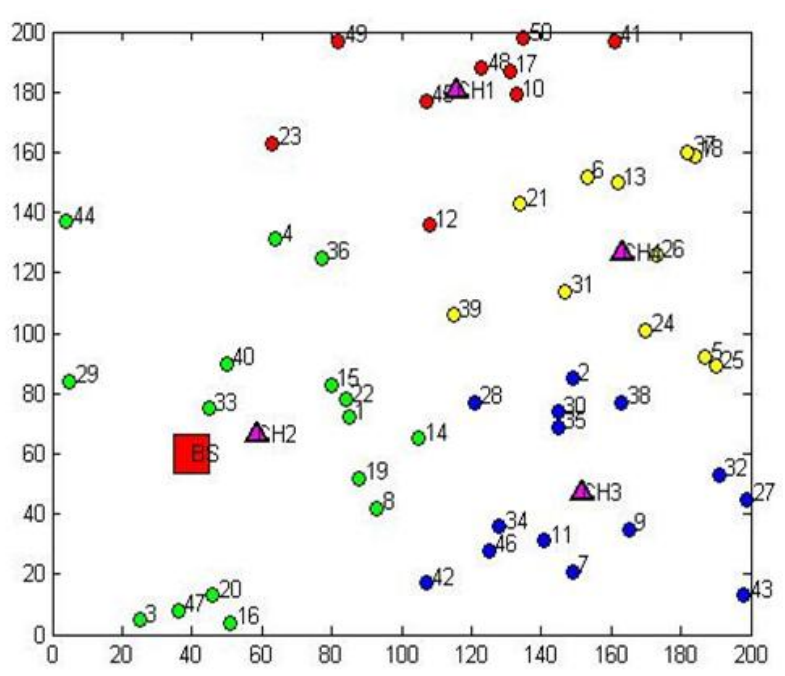

Fig 10: Deployment of Base station and clusters

\subsection{Number versus Energy}

Figure 11 describe about the Number of nodes versus Energy .In this figure we considered as X-label as number of node and Y-label as energy consumption. At the $10 \mathrm{~m}$ communication range twenty node will consume 56micro joules energy, 30 nodes consume 72 micro joules energy, 40 nodes consume 108 micro joules energy so on. For the $15 \mathrm{~m}$ communication range consumption of energy is 61micro joules, 77 micro joules, 113 micro joules so on. It shows that, as the number node increase energy consumption also increases. From this paper energy minimized.

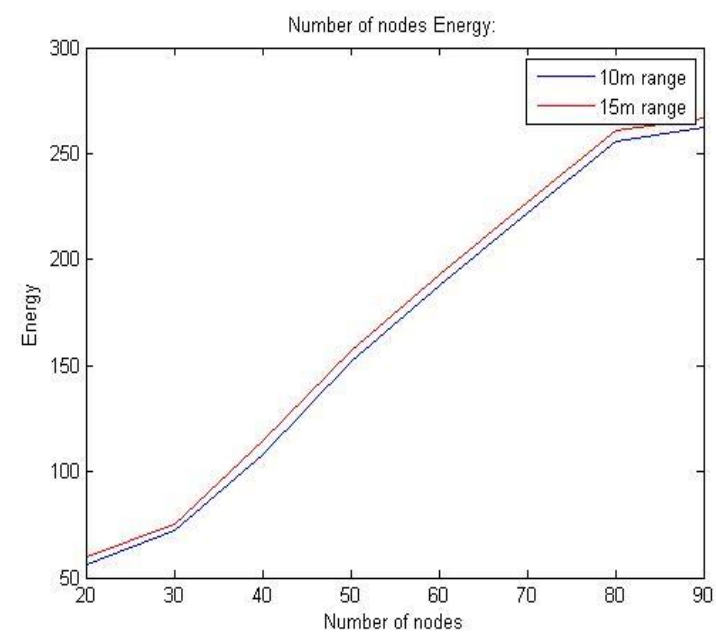

Fig 11: Number of nodes versus Energy

\subsection{Number of Node versus Delay}

Figure 12 describe about the Number of nodes versus Delay. In this figure we considered as $\mathrm{X}$-label as number of node and Y-label as delay. At10m communication range the twenty node will take time 10 micro second energy, 30 nodes 
will take time 16 micro second, 40 nodes will take time 108 micro second so on. For the $15 \mathrm{~m}$ communication range time taken is 15 micro joules, 21 micro joules, 113 micro joules so on It shows that, as the number node increase time consumption also more. From this paper minimized.

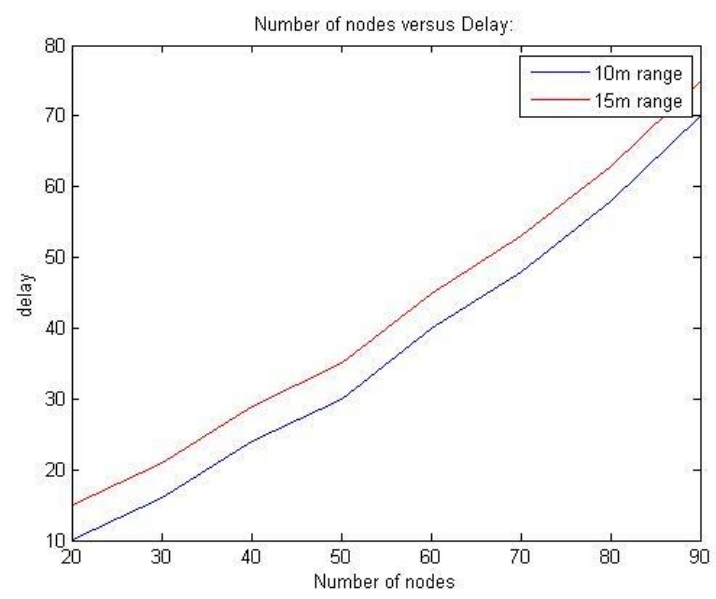

Fig 12: Number of nodes versus Delay

\subsection{Number of Node versus Faulty Node}

Figure 13 describe about the Number of nodes versus Faulty node. In this figure we considered as X-label as number of node and Y-label as Faulty node. At the $10 \mathrm{~m}$ communication range the twenty node there will be a 3 faulty node, 30 node there will be a 5 faulty node, for 40 nodes there will be 3 faulty node, so on. For the $15 \mathrm{~m}$ communication range Faulty nodes are $8,10,8$ so on. It shows that, as the number node increase Faulty node also increases. From this paper we reduce the number of faulty node.

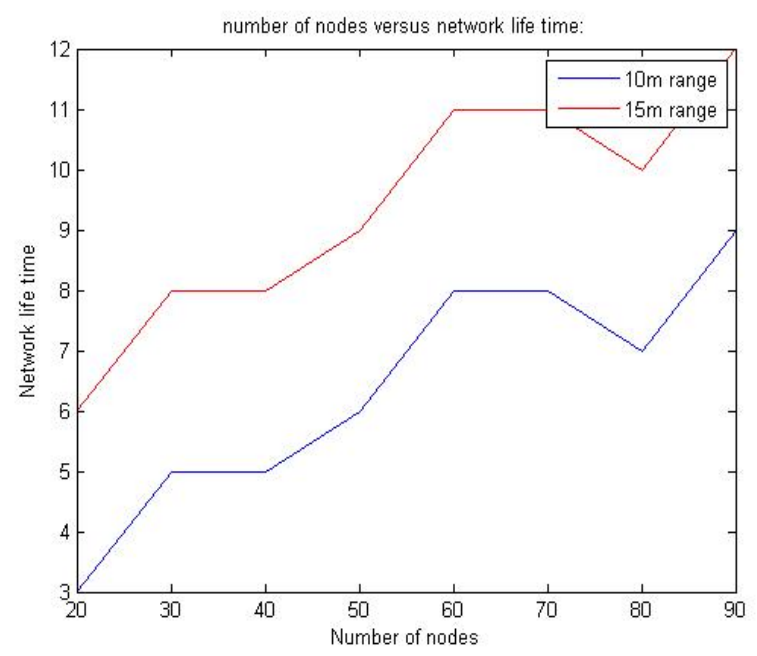

Fig 13: Number of nodes versus Faulty node

\subsection{Number of Node versus Network Life Time}

Figure 14 describe about the Number of nodes versus Network life time. In this figure we considered as X-label as number of node and Y-label as Network life time. .At the $10 \mathrm{~m}$ communication range Twenty node having the network life time is $2300 \mathrm{sec}, 30$ nodes having the network life time is $2500 \mathrm{sec}, 40$ nodes having the network life time is $3000 \mathrm{sec}$ so on. For the $15 \mathrm{~m}$ communication range Network life time will be $2800 \mathrm{sec}, 3000 \mathrm{sec}, 3500$ so on. It shows that, as the number node increase network life time also increase. From this paper network life time increases.

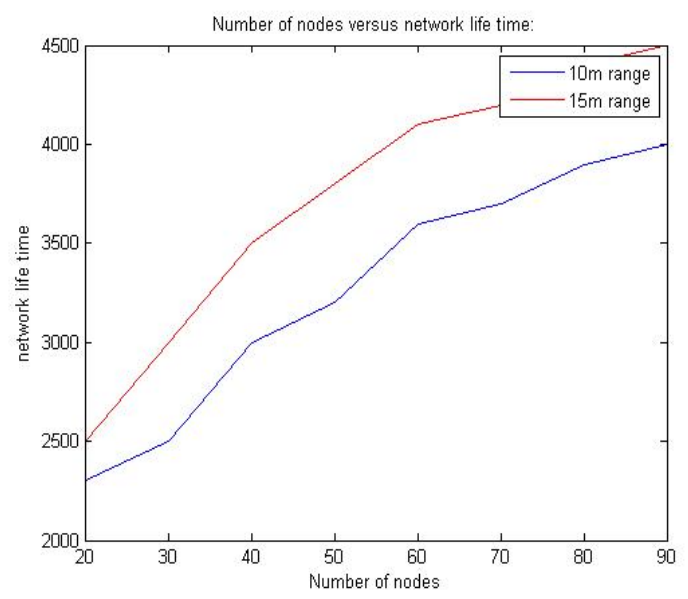

Fig 14: Number of nodes versus Network life time

\section{CONCLUSION}

A Sensor-Cloud gathers and process data from numerous sensor networks, facilitates data allocating on large scale, and cooperate with each others with the applications on cloud amongst customers. Sensor-Cloud facilitates customers to simply get together, right to use, procedure, visualize, investigate, accumulate, distribute.

In order to minimize problem of Resource management and Fault tolerance we grouped the resources in sensor cloud. As per user requirements the admin will send the data. Admin will check if there are fault nodes in sensor cloud, if admin found fault node then it will be removed. The result shows there is a proper management of resources without losing the accuracy of data and there is an enlarge in the network life time of the system and also faulty nodes are deleted and eliminated from the network without affecting the network.

\section{REFERENCES}

[1]. Akyildiz, I.; Su, W.; Sankarasubramaniam, Y.; Cayirci, E. A survey on sensor networks. IEEECommun. Mag. 2002, 40, 102-114.

[2]. Sensor-Cloud, http://sensorcloud.com/system-overview. [3]. C. O. Rolim, F. L. Koch, C. B. Westphall, J. Werner, A. Fracalossi, and G. S. Salvador, "A cloud computing solution for patient's data collection in health care institutions," in Proceedings of the 2nd International Conference on eHealth, Telemedicine, and Social Medicine (eTELEMED '10), pp. 95-99, February 2010.

[4]. Chi yang, chang liu, xuyun zhang, surya nepal, and jinjun chen, senior member, a time efficient approach for detecting errorsin big sensor data on cloud'. ieee transactions on parallel and distributed systems, vol. 26, no. 2 , february 2015 
[5]. Sudip Misra, Senior Member, IEEE, Subarna Chatterjee, Student Member, IEEE, and Mohammad S. Obaidat, Fellow, IEEEOn Theoretical Modeling of Sensor Cloud: A Paradigm Shift from Wireless Sensor Network.

[6]. Atif Alamri, Wasai Shadab Ansari, Mohammad Mehedi Hassan,A Survey on Sensor-Cloud: Architecture, Applications and Approaches

[7]. Subasish Mohapatra,K. Smruti Rekha Sensor-Cloud: A Hybrid Framework for Remote Patient Monitoring, International Journal of Computer Applications (0975 8887) Volume 55- No.2, October 2012.

[8]. Kian Tee Lan, (2010)," What's NExT? Sensor+Cloud?", in Proceeding of 7th International workshop on Data Management for Sensor networks, ACM Digital Library, ISBN: 978-1-4503-0416-0, 2010.

[9]. MadokaYuriyama, Takayuki Kushida, (2010), "Sensor Cloud Infrastructure: Physical Sensor Management with Virtualized Sensors on Cloud Computing" 13th International Conference on Network-Based Information Systems, IEEE, 2010

[10]. A. Rowe, V. Gupta, R. Raj Kumar, (2009), "Lowpower clock synchronization using electromagnetic energy radiating from AC power lines", The 7th ACM Conference on Embedded Networked Sensor Systems (SenSys), 2009.

[11]. S. R. Madden and M. J. Franklin, (2002), "Fjording the Stream: An Architecture for Queries over Streaming Sensor Data," The $18^{\text {th }}$ nternational Conference on Data Engineering, 2002.

[12]. R. Katsuma, Y. Murata, N. Shibata, K. Yasumoto and M. Ito, (2009), "Extending k-Coverage Lifetime of Wireless Sensor Networks Using Mobile Sensor Nodes," The 5th IEEE International Conference on Wireless and Mobile Computing, Networking and Communications(WiMob_2009), 2009.

[13]. J. Hill, R. Szewczyk, A. Woo, S. Hollar, D. Culler and K. Pister, (2000), "System architecture directions for networked sensors," International Conference on Architectural Support for Programming Languages and Operating Systems, 2000.

[14]. K. Matsumoto, R. Katsuma, N. Shibata, K. Yasumoto and M. Ito, (2009), "Extended Abstract: Minimizing Localization Cost with Mobile Anchor in Underwater Sensor Networks," The Fourth ACM International Workshop on Under Water Networks (WUWNet), 2009.

[15]. T. I. Sookoor, T. W. Hnat, P. Hooimeijer, W. Weimer and K. Whitehouse,(2009), "Macro debugging: Global Views of Distributed Program Execution," The 7th ACM Conference on Embedded Networked Sensor Systems (SenSys 2009), 2009.

[16]. J. Shneidman, P. Pietzuch, J. Ledlie, M. Roussopoulos, M. Seltzerand M. Welsh, (2004), "Hourglass: An Infrastructure for Connecting Sensor Networks and Applications," Harvard Technical Report TR-21-04, 2004.

[17]. Albert Alexe, R. Exhilarasie, (2011), "Cloud computing based Vehicle tracking Information Systems", IJCST, Volume 2, Issue 1, March 2011. 